\title{
Wide-field endoscopic submucosal dissection for the treatment of Barrett's esophagus neoplasia $\square$
}

\section{(ㄷ)(요 $\odot$}

\author{
Authors \\ Masami Omae ${ }^{1}$, Hannes Hagström, ${ }^{1,2}$ Nelson Ndegwa ${ }^{1,3}$, Michael Vieth ${ }^{4}$, Naining Wang ${ }^{5}$, Miroslav Vujasinovic ${ }^{1}$, \\ Francisco Baldaque-Silva ${ }^{1}$
}

Institutions

1 Department of Upper Gastrointestinal Diseases, Karolinska University Hospital and Karolinska Institute, Stockholm, Sweden

2 Clinical Epidemiology Unit, Department of Medicine, Solna, Karolinska Institutet, Stockholm, Sweden

3 Department of Medical Epidemiology and Biostatistics, Karolinska Institutet, Stockholm, Sweden

4 Institute of Pathology, Klinikum Bayreuth, Bayreuth, Germany

5 Department of Pathology, Karolinska University Hospital and Karolinska Institute, Stockholm, Sweden

submitted 25.4 .2020

accepted after revision 20.1.2021

Bibliography

Endosc Int Open 2021; 09: E727-E734

DOI 10.1055/a-1386-3668

ISSN 2364-3722

(C) 2021. The Author(s)

This is an open access article published by Thieme under the terms of the Creative Commons Attribution-NonDerivative-NonCommercial License, permitting copying and reproduction so long as the original work is given appropriate credit. Contents may not be used for commercial purposes, or adapted, remixed, transformed or built upon. (https://creativecommons.org/licenses/by-nc-nd/4.0/)

Georg Thieme Verlag KG, Rüdigerstraße 14,

70469 Stuttgart, Germany

Corresponding author

Francisco Baldaque-Silva, MD PhD, Department of Upper

Gastrointestinal Diseases, Karolinska University Hospital and Karolinska Institute, Huddinge, 14186 Stockholm, Sweden Fax: +4658580000

fbaldaquesilva@gmail.com

\section{ABSTRACT}

Background and study aims Implementation of endoscopic submucosal dissection (ESD) for the treatment of Barrett's esophagus neoplasia (BEN) has been hampered by high rates of positive margins and complications. Dissection with wider margins was proposed to overcome these problems, but was never tested. We aim to compare WideField ESD (WF-ESD) with conventional ESD (C-ESD) for treatment of BEN.

Patients and methods This was a cohort study of all ESDs performed in our center during 2011 to 2018. C-ESD was the only technique used before 2014, with WF-ESD used beginning in 2014. In WF-ESD marking was performed $10 \mathrm{~mm}$ from the tumor margin compared to $5 \mathrm{~mm}$ with C-E.

Results ESD was performed in 90 cases, corresponding to 74 patients, $84 \%$ male, median age 69 . Of these, 22 were C-ESD (24\%) and 68 were WF-ESD (76\%). The en bloc resection rate was 95 vs $100 \%$ (ns), the positive lateral margin rate was $23 \%$ vs $3 \%(P<0.01)$, the R0 rate was $73 \%$ vs $90 \%$, and the curative resection rate was $59 \%$ vs $76 \%$ in the C-ESD and WF-ESD groups, respectively, (both $P>0.05$ ). The procedure speed was 4.4 and $2.3(\mathrm{~min} / \mathrm{mm})$ in the C-ESD and WF-ESD groups $(P<0.01)$, respectively. WF-ESD was associated with less post-operative strictures, $6 \%$ vs $27 \%$ ( $P=$ 0.01 ), with no local recurrence but no significantly reduced risk of metachronous recurrence (Hazard Ratio $=0.46$, 95\% $\mathrm{Cl}=0.14-1.46)$, during a follow-up of 13.4 and 9.4 months in the C-ESD and WF-ESD cohorts, respectively.

Conclusions WF-ESD is associated with a reduction in positive lateral margins, faster dissection, and lower stricture rates. Further prospective, multicenter studies are warranted to evaluate its role in clinical practice.

\section{Introduction}

Barrett's esophagus (BE) is an established risk factor for the development of esophageal adenocarcinoma (EAC) [1]. Endoscopic treatment of early Barrett's neoplasia is associated with increased survival and has a good safety profile [2]. Current guidelines recommend endoscopic resection of visible lesions on Barrett's mucosa and ablation of the remaining BE due to risk of synchronous or metachronous neoplasia in $20-41 \%$ of patients [3,4].

Endoscopic mucosal resection (EMR) enables en bloc resection in lesions up to 15 to $20 \mathrm{~mm}$ along the gastrointestinal 
tract. Larger lesions are usually resected with piecemeal EMR that hampers proper histological assessment and increases the risk for residual lesions and recurrence.

In most centers, endoscopic resection of Barrett's neoplasia is performed with EMR due to its safety profile and feasibility in an outpatient setting. The current strategy of EMR followed by ablation is associated with complications in up to $12 \%$ of cases, including pain (3\%), bleeding (1.6\%), stricture $(5 \%-12 \%)$ and perforation (0.6\%) [5,6] Additionally, buried glands can grow beneath the neosquamous epithelium after ablation [7]. Endoscopic submucosa dissection (ESD) was developed for en bloc resection of early gastrointestinal neoplasms and its role is established in superficial esophageal squamous cell neoplasia, early gastric neoplasia and some colorectal lesions [8]. The role of ESD in the treatment of BE neoplasia has been studied in smaller series with suboptimal results, mainly due to positive lateral margins and few studies compare ESD with EMR in this setting [9]. Most previous studies were performed in the West where ESD experience is lower, and the high rate of positive lateral margins may reflect poor delineation of the lesion or suboptimal ESD technique [10]. In fact, even in the most specialized centers, with the use of different advanced endoscopic modalities, full identification, characterization and delineation of neoplastic BE is suboptimal $[11,12]$. Higher costs of ESD, mainly associated with longer hospital admission, have also hampered its use in BE. However, it was recently demonstrated that ESD can be safely performed in an outpatient setting, reducing costs and hospitalization risks [13].

Some authors have advocated a new approach for ESD on BE, based on wider resection margins $(5-10 \mathrm{~mm})$, margins even wider than those described for wide-field colorectal EMR [14]. This strategy aims to decrease the rate of positive lateral margins, improving curability and decreasing recurrence. This approach has been advocated by others, but never tested [15].

The aim of this study was to evaluate the feasibility, safety, and efficacy of wide-field ESD (WF-ESD) for resection of BE neoplasia, comparing it to conventional ESD (C-ESD).

\section{Patients and methods}

This was a cohort study from a prospectively collected database of all patients with neoplastic BE that were treated with ESD between June 2011 and June 2018 at the Karolinska University Hospital in Stockholm, Sweden. This study was approved by the Stockholm Regional Ethical Committee (2020-05737).

Our hospital is a national reference center for the management of esophageal neoplasias in Sweden. In our center, endoscopic resection of neoplastic Barrett's esophagus with ESD started in 2011. Due to early suboptimal results caused by positive lateral margins in some cases, as previously described in the literature [16], we decided to start performing WF-ESD, for the resection of all Barrett's neoplastic lesions from January 2014. The indications for ESD were the presence of a visible lesion on Barrett's mucosa with well differentiated neoplasia on biopsy specimens that engaged the mucosa or superficial submucosa $(\mathrm{Sm} 1,<500 \mu \mathrm{m})$ and with no ulceration. All cases were discussed in a multidisciplinary team (MDT) conference before the ESD procedure.

All patients received a detailed explanation regarding the procedure, its risks, benefits, and alternatives and provided informed consent. Participants' clinical and demographic information was obtained and recorded at enrollment. Patients under antiplatelet drugs or anticoagulants were handled according to published guidelines.

\section{Endoscopic assessment}

All Barrett's mucosa underwent careful endoscopic assessment with high resolution endoscopy (GIF-HQ190]; Olympus, Hamburg, Germany) with the corresponding transparent hood (D201-11804; Olympus). Barrett's mucosa was classified according to the Prague criteria and evaluated using white light, narrow band imaging and chromoendoscopy with acetic acid $1.5 \%$, in overview and near focus mode. All visible lesions on BE mucosa were characterized according to the Paris classification and registered accordingly.

\section{ESD technique}

All ESDs were performed under full anesthesia and orotracheal intubation. Electrosurgical current was applied using a standard electrosurgical generator (VIO300 D, ERBE, Tübingen, Germany). Tumors were characterized and delineated using narrow band imaging (NBI), acetic acid and magnification endoscopy. After carefully evaluation, circumferential marking was performed around the tumor using a Dual knife (KD-650 L, Olympus). In conventional ESD, marking was performed up to $5 \mathrm{~mm}$ from the tumor margins. In the WF-ESD group, marking was performed approximately $10 \mathrm{~mm}$ from the tumor margins

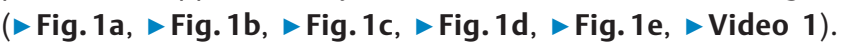
Hyaluronic acid (Sigmavisc, Life Partners, Europe) was then injected into the submucosal layer to lift the mucosa. An initial incision was made with the Dual knife on the distal side followed by an incision on the proximal side ( $\triangleright$ Fig. 1f). The tumor was then completely removed by submucosal dissection ( $\triangleright$ Fig. 1g). Coagrasper (FD-411QR, Olympus) was used for hemostasis during the procedure or to coagulate vessels present in the submucosal layer. After resection of the specimen, a carefully interrogation of the post ESD ulcer was performed in order to detect muscular tears or bleeding vessels. Resected specimens were mounted on boards with pins and fixed in $10 \%$ formalin for 24 hours ( $>$ Fig. 1h). After fixation, all resected specimens were cut into longitudinal $2 \mathrm{~mm}$ slices in width and then embedded in paraffin and stained with hematoxylin-eosin.

\section{Assessment after ESD}

Patients were under continuous monitoring for 4 hours after the procedure and kept nil per os until the following day. Intravenous proton pump inhibitors (PPI) were administered in the first 24 hours, followed by high-dose of oral PPI for 8 weeks. From 2014 (when all patients were treated with WF-ESD) oral steroids were prescribed in all cases with resections larger than 3/4 of the luminal circumference, according to published literature [17]. 


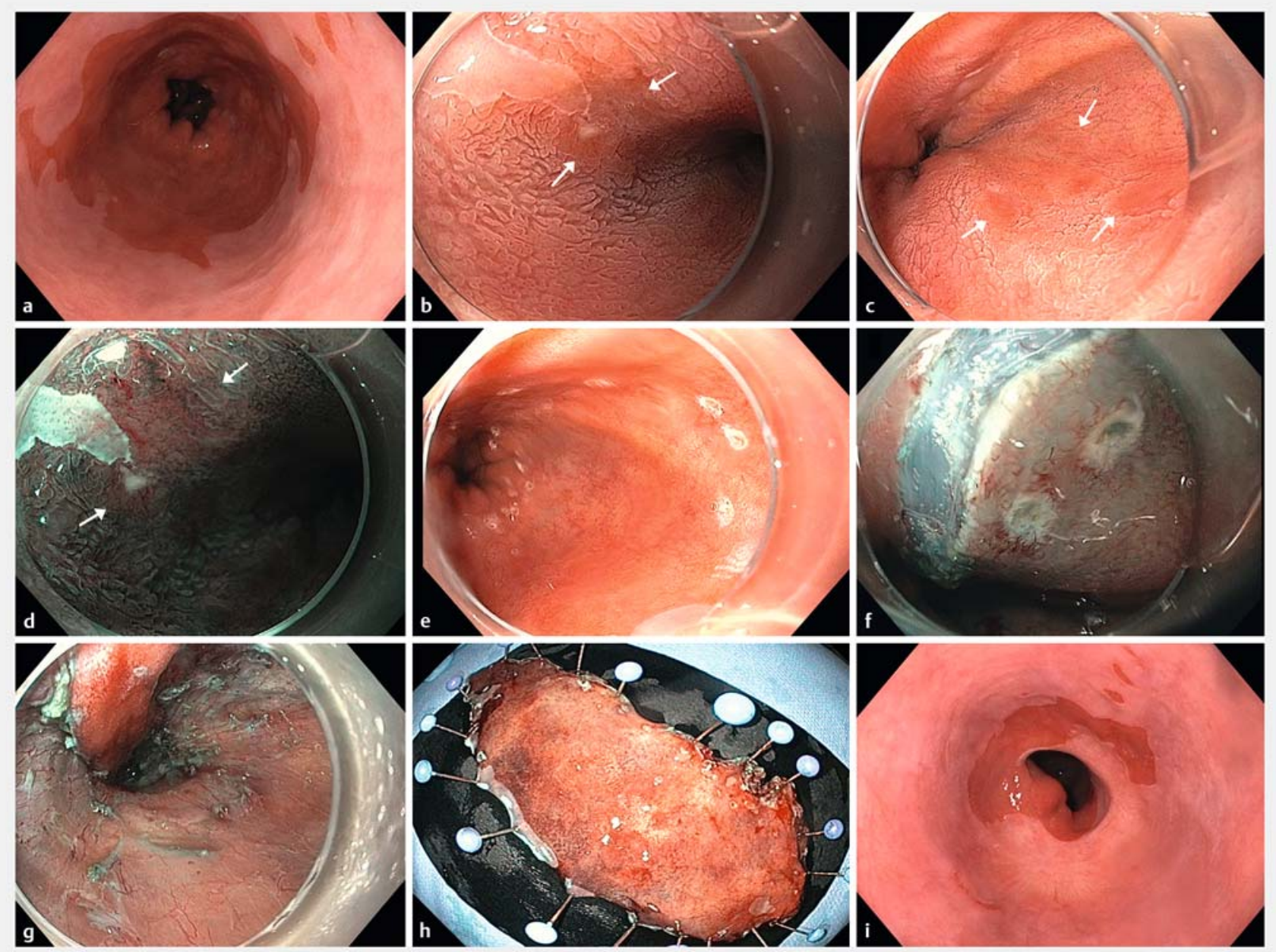

- Fig. 1 a White light endoscopy of a long-segment (C3M9) Barrett's esophagus (BE) with multifocal high-grade dysplasia (HGD). Visible lesions were not detected by white light endoscopy. $\mathbf{b}, \mathbf{c}$ White light endoscopy with acetic acid chromoendoscopy showing multifocal lesions with loss of acetowhitening. $\mathbf{d}$ Magnified endoscopy with narrow band imaging showing irregular vascular and mucosal pattern. e Marking with wider (10-mm) free margins with dual knife. $\mathbf{f}$ Incision outside the marking dots. $\mathbf{g}$ Endoscopic submucosal dissection (ESD) was performed with resection of $4 / 5$ of the esophageal circumference along $9 \mathrm{~cm}$. h Resected specimen with $9.5 \times 5.0 \mathrm{~cm}$ in size. Pathology assessment revealed the presence of Barrett's mucosa with multifocal low and high-grade dysplasia, with free margins (R0) and no lymphovascular invasion. i Follow-up endoscopy at 3 months with mucosal healing, neosquamous epithelium and neither strictures nor macroscopic lesions. Distal esophagus/cardia with similar width as before ESD.

Upper endoscopy was performed 2 to 3 weeks after ESD to detect and treat strictures if they occurred. Afterwards, conventional follow-ups with high-definition chromoendoscopy were performed at 2, 6, and 12 months and then each year in the case of curative resection ( $\mathbf{F i g . 1 i}$ ). In the case of noncurative resection or recurrence, the case was discussed again at an MDT conference. Signs or symptoms of complications, readmission, transfusion requirements and interventions were checked at the 2-3 weeks and at 2 months assessments. Follow-up was defined as the period between the ESD and the first following endoscopic or surgical treatment, or between the ESD and last endoscopic follow-up (in patients without interventional treatment after ESD).

\section{Outcome parameters}

The primary outcomes were the rates of en bloc, R0, curative resection, recurrence and procedure time. The secondary outcomes were adverse events, namely bleeding, perforation, stricture, and need for unplanned medical assistance after discharge.

\section{Primary outcome parameters}

We assessed the outcomes of C-ESD and WF-ESD, i. e., before and after January 2014. All histologic specimens were reviewed by two dedicated gastrointestinal pathologists and assessment was based on the Vienna classification [18]. En bloc resection was defined as excision of the target lesions in a single specimen. In up to $82 \%$ of Barrett's patients with high-grade dysplasia/carcinoma, the dysplastic lesions are multifocal $[19,20]$, 


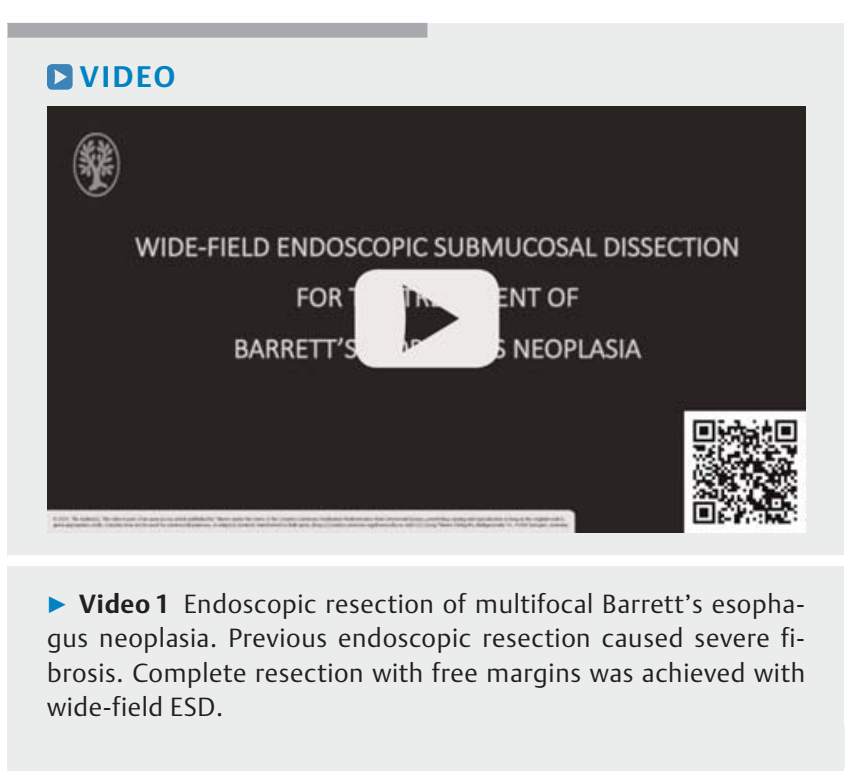

being the size assessment of neoplastic lesions difficult to evaluate and compare in this context. For that reason, we decided to assess the size of the resected specimens and not the size of the different neoplastic lesions.

$\mathrm{R} 0$ or radical resection was defined as the presence of negative lateral (LM) and vertical margins (VM) for high-grade dysplasia/cancer in the ESD specimens as described before [15]. Non-radical resection corresponded to R1 resections.

Curative resections were the $\mathrm{R} 0$ resections of well/moderately differentiated neoplasias that engaged the mucosa or superficial submucosa ( $\mathrm{Sm} 1$ ) and had no lymphovascular involvement. Non-curative resections were resections in which one of those parameters were not fulfilled [8].

The duration of ESD was defined as the time (in minutes) from the first incision until retrieval of the specimen. A velocity index of time ( $\mathrm{min})$ divided by the resected size $(\mathrm{mm})$ was calculated in all ESDs.

Local recurrence was defined as high-grade dysplasia/cancer detected within $2 \mathrm{~cm}$ of the previous ESD scar [15] and a metachronous recurrence was defined as neoplasia located more than $2 \mathrm{~cm}$ away from the ESD scar [15], at any of the follow-up endoscopies, before ablation treatment. Complete remission of neoplasia was defined as the absence of suspicious lesions on gastroscopy with ME-NBI and chromoendoscopy with acetic acid and no histologic evidence of dysplasia/cancer applying the Seattle protocol on the follow-up endoscopies [15]. After removal of all visible lesions and when curative resection was achieved, patients were treated systematically with radiofrequency ablation for complete Barrett eradication, in all patients in both cohorts.

\section{Secondary outcome parameters}

Adverse events were defined as any complication in which ESD or related procedures (such as anesthesia) were a contributing factor and included bleeding, perforation, stricture and death.

Perforation was defined as a full thickness breach in the esophageal wall with or without symptoms. Intraprocedural bleeding that led to blood transfusion or premature termina- tion of endoscopic resection was considered a complication. Delayed bleeding was defined as bleeding up to 2 weeks after the procedure that required presentation to the emergency department, hospital admission or any medical intervention. An esophageal stricture was defined as a narrowing of the esophageal lumen to the point where it was not possible to pass through with a standard $9.9 \mathrm{~mm}$ diameter gastroscope [21]. ESD related mortality was considered as death up to 30 days after the procedure in which the ESDs or any related procedures were a contributing factor.

\section{Statistical analysis}

Demographic and baseline characteristics were summarized as medians with interquartile ranges (IQR) for continuous data, and as total numbers and percentages for categorical data. Differences between C-ESD and WF-ESD were analyzed using the Mann-Whitney U test or Fischer's exact test as appropriate. Univariable logistic or Cox regression was used to obtain and hazard ratios (HR) for selected outcomes. We did not perform multivariable regression due to few outcomes and inherent risk of overfitting [22]. Statistical analyses were performed using SPSS, version 20 (SPSS Japan Inc, Tokyo, Japan) and STATA version 13.0 (Stata Corp, College Station, Texas, United States). $P<0.05$ was considered as statistically significant.

\section{Results}

\section{Patient characteristics}

A total of 74 patients, corresponding to 90 neoplastic Barrett's lesions, 84\% male, median age 69 (IQR 63-74), fulfilled the inclusion criteria and were treated with ESD. Of the 90 ESDs, 22 cases corresponded to C-ESD (24\%) and 68 to WF- ESD (76\%). Regarding previous treatment, three of 22 in C-ESD cohort and 17 of 68 in the WF-ESD cohort had previous endoscopic treatment. There were no significant differences between the two groups of patients in terms of gender, age and Barrett's circular and maximal lengths (all $P>0.05$ ) ( $\triangleright$ Table 1 ). No significant differences were observed between the two cohorts in terms of location $(P>0.05)$, but macroscopic type was significantly different between groups, with elevated/flat/depressed morphology present in 19/3/0 vs 29/29/10 of cases in C-ESD and WFESD groups, respectively $(P<0.01)$.

\section{Primary outcomes}

En bloc resection rate was 95 vs $100 \%$, R0 rate was 73 vs $90 \%$ and curative resection rate was 59 vs $76 \%$ in C-ESD and WFESD groups, respectively $(P>0.05)$. The percentage of the circumference surface resected, was $<25 \%, 25 \%$ to $50 \%, 50 \%$ to $75 \%$, and $>75 \%$ in $14 \%, 45 \%, 27 \%$, and $14 \%$ and in $3 \%, 35 \%$, $40 \%$, and $22 \%$ of cases in the c-ESD and WF-ESD groups, respectively. The median procedure time was 147 (range 32480 ) and 96 (range 15-388) minutes in C-ESD and WF- ESD $(P$ $<0.05)$, corresponding to a velocity of 4.4 and $2.3(\mathrm{~min} / \mathrm{mm})(P$ $<0.01$ ), respectively ( $>$ Table 2 ). There were no significant differences in histology of the resected specimens in both groups (all $P>0.05$ ). Positive lateral margins were less likely in WF-ESD $(2 / 68,3 \%)$ compared to C-ESD $(5 / 22,23 \%)(P<0.01)$, while 
- Table 1 Patient and lesions characteristics in conventional and wide-field endoscopic submucosal dissection (ESD), $n=90$.

\begin{tabular}{|c|c|c|c|}
\hline & Conventional ESD $(n=22)$ & Wide-field ESD $(n=68)$ & $P$ value \\
\hline Age (years, median, IQR) & $68(66-73)$ & $70(63-74)$ & 0.62 \\
\hline Sex (male, n/\%) & $16(73 \%)$ & $60(88 \%)$ & 0.10 \\
\hline \multicolumn{4}{|l|}{ Length of extension of BE } \\
\hline Short segment/long segment BE (n, \%) & $8 / 14(63 \%)$ & $26 / 42(61.8 \%)$ & 0.88 \\
\hline Circular extent (length of ' $C$ ') (cm, median, IQR) & $1(0-4)$ & $1(0-4)$ & 0.89 \\
\hline Extent of tongue (length of ' $\mathrm{M}$ ') ( $\mathrm{cm}$, median, IQR) & $4(2-5)$ & $3(1-8)$ & 0.92 \\
\hline \multicolumn{4}{|l|}{ Location } \\
\hline Junction/thoracic esophagus & $20 / 2(9 \%)$ & $68 / 14(21 \%)$ & 0.34 \\
\hline \multicolumn{4}{|l|}{ Tumor morphology } \\
\hline Elevated/flat/depressed type & $19 / 3 / 0$ & $29 / 29 / 10$ & 0.001 \\
\hline \multicolumn{4}{|l|}{ Histology of biopsy specimen } \\
\hline LGD/HGD/EAC & $1 / 14 / 7$ & $7 / 35 / 26$ & 0.71 \\
\hline
\end{tabular}

- Table 2 Lesion characteristics in conventional and wide-field endoscopic submucosal dissection (ESD), $n=90$.

\begin{tabular}{|c|c|c|c|}
\hline & Conventional ESD $(n=22)$ & Wide-field ESD $(n=68)$ & $P$ value \\
\hline Mean resected size (mm, IQR) & $40(30-70)$ & $44(35-60)$ & 0.27 \\
\hline $\begin{array}{l}\text { Resected circumferential lumen } \\
<25 \% / 25 \%-50 \% / 50 \%-75 \% />75 \%\end{array}$ & $3 / 10 / 6 / 3$ & $2 / 24 / 27 / 15$ & 0.18 \\
\hline $\begin{array}{l}\text { Histology of ESD specimens } \\
\text { LGD/HGD/EAC }\end{array}$ & $2 / 6 / 14$ & $6 / 30 / 32$ & 0.37 \\
\hline $\begin{array}{l}\text { Tumor differentiation (EAC) } \\
\text { G1-2/G3 }\end{array}$ & $21 / 1$ & $64 / 4$ & 1.0 \\
\hline $\begin{array}{l}\text { Tumor depth (EAC) } \\
\mathrm{M}-\mathrm{SM} 1 />\mathrm{SM} 2\end{array}$ & $18 / 4$ & $62 / 6$ & 0.25 \\
\hline Positive lateral margin & $5(23 \%)$ & $2(3 \%)$ & 0.009 \\
\hline Positive vertical margin & $2(9 \%)$ & $6(9 \%)$ & 1.0 \\
\hline Positive lymphatic invasion & $2(9 \%)$ & $8(12 \%)$ & 1.0 \\
\hline Positive vascular invasion & $5(23 \%)$ & $11(16 \%)$ & 0.53 \\
\hline $\begin{array}{l}\text { Metachronous recurrence } \\
\text { Negative/positive }\end{array}$ & $16 / 6$ & $62 / 6$ & 0.04 \\
\hline $\begin{array}{l}\text { Local recurrence } \\
\text { Negative/positive }\end{array}$ & $20 / 2$ & $68 / 0$ & 0.06 \\
\hline
\end{tabular}

there was no difference in respect to the vertical margin (2/22 $(9 \%)$ in the C-ESD vs six of $68(9 \%)$ in the WF-ESD groups, $(P=$ 1.0).

All patients were followed in the outpatient clinic. The mean follow-up in the C-ESD cohort was 13.4 (SD 15) months and the in the WF-ESD cohort was 9.4 (SD 9.9) months, $(P=0.27)$. Local recurrence was present in $2 / 22(9 \%)$ and in none of $68(0 \%)$ of the cases in the C-ESD and WF-ESD groups, respectively ( $P>$ 0.05). The Cox regression shown no significant differences between the groups in terms of metachronous recurrence (hazard ratio $0.46,95 \% \mathrm{Cl}=0.14-1.46, P=0.19$ ).

Regarding previous endoscopic treatment, three of 22 in the C-ESD group had previous treatment, and metachronous lesions were found in two of three of those vs four of 19 in those 
without previous treatment $(P=0.1)$. In the WF-ESD group, 17 of 68 had previous treatment, of those there was metachronous recurrence in four of 17 vs only two of 51 of those with no previous treatment $(P=0.01)$. There was no difference in the proportion of multifocal lesions between the two cohorts $(P=0.51)$.

In both groups, all but one case with recurrence were successfully treated with a new ESD resection. There were nine cases (41\%) of non-curative resection in the C-ESD cohort: eight were treated surgically and only one of those had lymph node metastasis in the surgical specimen. During follow-up, five of those patients died, one due to lung metastasis and the others due to unrelated causes. One 68 years-old male patient from the C-ESD group had lung metastasis and died 3 months after ESD. He had a C1M4 BE with high-grade dysplasia on the biopsy specimens. The ESD specimen showed the presence of well differentiated adenocarcinoma (invading the muscularis mucosae) R1, L0V0.

There were sixteen cases (24\%) of non-curative resection in the WF-ESD cohort: six were treated surgically and two of those had lymph node metastasis in the surgical specimen. During follow-up, three patients died, one due to peritoneal metastasis and the others due to unrelated causes. One 74 years-old male patient from the WF-ESD group had C10M11 BE with adenocarcinoma. The ESD specimen showed the presence of a well differentiated adenocarcinoma (pT1a), R0, L1V1. He died 17 months after ESD due to peritoneal metastasis.

\section{Secondary outcomes}

There was one case of bleeding and one case of perforation, both in the WF-ESD group and both were treated conservatively. Strictures were less frequent in the WF-ESD group, four of 68 (6\%) vs the C-ESD group, six of $22(27 \%)(P=0.01)$. In large resections, that engaged $>75 \%$ of the luminal circumference, strictures were more frequent in the C-ESD group (3/3, $100 \%)$ than in WF-ESD group 4/15 (27\%), $P=0.04$. The later received oral steroids prophylactically ( $>$ Table 3 ).

In the WF-ESD cohort, depth of invasion (presence of deep submucosal cancer relative to mucosal cancer) was the only identifiable factor for non-radicality (OR 60.0, 95\% confidence interval $[\mathrm{Cl}]$ 10.0-361.0, $P<0.001$ ). Lesion histology (presence of $E A C$ relative to absence of adenocarcinoma) was the only factor associated with non-curability (OR 21.0 95\% Cl 14.5-97.2, $P<0.001)$. Resection of more than $75 \%$ of the circumferential lumen was the only identifiable factor for the presence of complications, namely strictures (OR 6.4 95\% Cl 1.4-29.1 $P<0.02$ ).

\section{Discussion}

We found that wide-field ESD for the treatment of BE neoplasia, is associated with reduction of tumor-positive lateral margins and reduction of local recurrence. Wide-field ESD also enables a faster dissection and is associated with a lower stricture rate, although that might be a consequence of steroid prophylaxis that was introduced together with the WF-ESD protocol.

In Barrett's neoplasia, endoscopic resection is recommended when lesions are limited to the mucosa or superficial submucosa, are well or moderately differentiated and have no lymphatic or vascular invasion. These cases harbor a low risk of lymph node metastasis (1.4\%) and endoscopic treatment is considered curative. European and American guidelines recommend EMR as the first treatment for visible Barrett's neoplasia $[23,24]$. Using the standard therapy with EMR plus ablation, $2.4 \%$ of patients do not respond to therapies and $4 \%$ have recurrence of neoplasia [25]. Those limitations lead our center to adopt a resection strategy of Barrett's neoplasia based on en bloc resection, similar to the one widely used for gastric lesions. ESD enables en bloc resection of lesions $>20 \mathrm{~mm}$, as reflected in a recent meta-analysis showing that the mean specimen size with EMR is $20.4 \mathrm{~mm}$ compared to $37 \mathrm{~mm}$ in ESD $(P<$ 0.001 ) [26]. In our center and since 2011, all neoplastic lesions larger than $15 \mathrm{~mm}$ were resected en bloc with ESD irrespective of grade of dysplasia, after MDT conference. Later, the remaining Barrett's mucosa is ablated with RFA. In our study, it was possible to resect specimens with median size of $44 \mathrm{~mm}$ using WF-ESD. In comparison to C-ESD, WF-ESD was associated with a significant increase in the rate of free lateral margins. The fact that there was a tendency for larger resections with WF-ESD,

- Table 3 Study endpoints and technical results in conventional and wide-field endoscopic submucosal dissection (ESSD endoscopic submucosal dissection (ESD), $n=90$.

\begin{tabular}{|l|l|l|l|}
\hline & Conventional ESD (N=22) & Wide-field ESD (N=68) \\
\hline En bloc resection rate (\%) & $21(95 \%)$ & $68(100 \%)$ & 0.24 \\
\hline R0 resection rate (\%) & $16 / 22(72.7)$ & $61 / 68(89.7)$ & 0.08 \\
\hline Curative resection rate (\%) & $13 / 22(59.1)$ & $52 / 68(76.5)$ & 0.17 \\
\hline Procedure time (min, median, IQR) & $147(88-295)$ & $96(77-164)$ & 0.04 \\
\hline time (min) / resected size (mm) (median, IQR) & $4.4(2.5-6.6)$ & $2.3(1.7-3.6)$ & 0.003 \\
\hline Complications overall (\%) & $6(27 \%)$ & $6(9 \%)$ & 0.06 \\
\hline Bleeding (\%) & $0(0 \%)$ & $1(1.5 \%)$ & $1(1.5 \%)$ \\
\hline Perforation (\%) & $0(0 \%)$ & $4(6 \%)$ \\
\hline Stricture (\%) & $6(27 \%)$ & 1.0 \\
\hline
\end{tabular}


but not statistically significant, may be explained by the detection of smaller lesions by the referring centers, due to the advances in endoscopic technologies and increasing awareness of the referring endoscopists with mapping of Barrett's mucosa, along the study period. Another advantage of ESD is that it enables resection of lesions with submucosal fibrosis, being EMR difficult in that context. Different causes may be associated with submucosal fibrosis on BE, namely esophagitis, previous biopsies or endoscopic treatment. Fibrotic lesions were excluded from most studies on BE ESD, but have recently been associated with a slight decreased rate of R0 and curative resections [15]. Despite the large size of the lesions, the presence of deep fibrosis due to previous endoscopic treatment in 17 of 68 cases and the presence of deep submucosal invasion in six of 68 cases, WF-ESD was feasible in all, with en bloc resection rate of $100 \%$, and R0 and curative resection in 61 of 68 (90.0\%) and in 52 of 68 cases (76\%), respectively. These are the highest rates reported so far in long segment BE [27]. Considering just fibrotic lesions, WF-ESD enabled en bloc, R0 and curative resection in 17 of 17,15 of 17 and in 14 of 17 of the cases. Deep submucosal invasion has been associated with decreased R0 rates [15]. That was the case in our series in which deep lesions $(\geq \mathrm{SM} 2)$ were present in six of 68 cases, contributing to six of seven of the $\mathrm{R} 1$ resections.

There are some factors that would hamper the use of WFESD namely high complications rate and longer procedure time. The stricture rate for C-ESD was $27 \%$ and higher than some of previous reports in the field. That might be explained by the size of the resected specimens that were larger than in those studies. The stricture rate with WF-ESD (6\%) is lower than most previously reported series of C-ESD. These results might be surprising considering the large resected areas (median size of $44 \mathrm{~mm}$, and engagement >3/4 circumference in 15 of 68 cases) and the presence of severe fibrosis in $25 \%$ (17/68) of WF-ESD cases. The low stricture rate may be related with the systematic use of oral steroids after WF-ESD engaging $>75 \%$ of the luminal circumference. First reports of high stricture rates after esophageal ESD were followed by recent rates as low as $2 \%$ (mostly after prophylaxis with oral steroids) [17]. Oral steroids have been used in several squamous cell carcinoma ESD series from Japan, but not reported in the largest BE ESD series from the West $[15,28]$. Another reason for the lower stricture rate might be the use of hyaluronic acid for submucosal lifting. Hyaluronic acid has anti-inflammatory, anti-fibrotic and regenerative proprieties and we used it in all patients [29]. Due to its high costs, it is seldom used for BE ESD. In fact, hyaluronic acid was not used in series with reported high stricture rates after BE ESD/EMR [27,29-31]. All four cases of esophageal stricture in the WF-ESD group had near circumferential ESD, with endoscopic treatment successful in them. In three patients, patency was achieved with balloon dilation and in the other, seven sessions of balloon dilation followed by temporary stent placement were needed for patency. With WF-ESD the rates of bleeding and perforation were both $1.9 \%$, making those rates similar to the ones reported in the biggest series on C-ESD for BE $[15,28]$. This reassures the safety of this technique in the appropriate setting. The procedure length is one of the factors that hampers the dissemination of the ESD technique. In our study, WF-ESD procedure time was shorter than that of the C-ESD procedure, and shorter than previously published ESD series [15, 16, 27, 28, 32]. Endoscopists engaged on ESD procedures (FBS, MO) had extensive experience on ESD with a total of more than 500 previous ESDs) previous to this study. Part of these difference on procedure length may be explained by improvement in techniques such as tunneling, along the study. With C-ESD, high caution should be taken with the lesions' margins and the maneuverability of the endoscope and knife may be limited due to the small fragment sizes. With wider margins, dissection can be faster and auxiliary techniques such as tunneling be more easily performed, especially in cases with fibrosis/submucosal invasion.

Most patients underwent endoscopic or surgical treatment after the ESD. Those treatments reduce or eliminate the remaining Barrett's mucosa and consequently the risk for metachronous recurrence. For that reason, we used a strict definition of follow-up, considering the period between the ESD and following surgical or endoscopic treatment (in treated patients). Using the Cox regression, it was possible to detect a trend for lower risk for metachronous recurrence in the WFESD cohort, but there were no significant differences between groups. This might be the result of an underpowered analysis due to the big pool of patients with surgical or endoscopic treatment after the ESD, in both cohorts. This analysis should be replicated in further studies in the field.

There are limitations to this study. The study was limited by its uncontrolled design, but it includes all the consecutive BE patients treated with ESD, and the switch from C-ESD to WFESD was well demarked in time, reducing the risk of selection bias. This was a single-center study and ESDs were conducted by endoscopists with significant experience with the technique before entering the study. Therefore, our results might not be generalizable to other endoscopy centers. The lower rate of strictures in the WF-ESD group might be associated with the introduction of steroid prophylaxis that did occur with initiation of WF-ESD. Endoscopic, surgical or chemoradiotherapy treatment after ESD have strong impact on the recurrence of neoplasia. In order to avoid bias, we used a stricter definition of follow-up than in previous published studies. That caused a reduction of the follow-up period. So, results on metachronous recurrence should be interpreted with caution, considering the short follow-up periods in both groups. Finally, it is reasonable to assume that besides endoscopists' experience, there is always a learning curve in terms of diagnostic and therapeutic endoscopy that might have influenced the outcomes.

\section{Conclusions}

In summary, WF-ESD is associated with favorable clinical and technical outcomes comparing with our previous results and published studies of C-ESD. High en bloc resection in WF-ESD can provide a more precise histologic assessment and favorable lateral R0 is associated with reduced local recurrence. Furthermore, WF-ESD enables a faster dissection with high safety profile. Overall, these results suggest that WF-ESD may be an op- 
tion in cases of BE neoplasia. Further prospective, multicentric studies are warranted in order to evaluate the role of WF-ESD in clinical practice, preferably using randomized, controlled trial designs.

\section{Competing interests}

The authors declare that they have no conflict of interest.

\section{References}

[1] de Jonge PJ, van Blankenstein M, Grady WM et al. Barrett's oesophagus: epidemiology, cancer risk and implications for management. Gut 2014; 63: 191-202

[2] Pech O, May A, Manner $\mathrm{H}$ et al. Long-term efficacy and safety of endoscopic resection for patients with mucosal adenocarcinoma of the esophagus. Gastroenterology 2014; 146: 652-660.e1

[3] Pech O, Behrens A, May A et al. Long-term results and risk factor analysis for recurrence after curative endoscopic therapy in $349 \mathrm{pa}-$ tients with high-grade intraepithelial neoplasia and mucosal adenocarcinoma in Barrett's oesophagus. Gut 2008; 57: 1200-1206

[4] Ell C, May A, Gossner L et al. Endoscopic mucosal resection of early cancer and high-grade dysplasia in Barrett's esophagus. Gastroenterology 2000; 118: 670-677

[5] Bulsiewicz W], Kim HP, Dellon ES et al. Safety and efficacy of endoscopic mucosal therapy with radiofrequency ablation for patients with neoplastic Barrett's esophagus. Clin Gastroenterol Hepatol 2013; 11: 636-642

[6] Qumseya BJ, Wani S, Desai M et al. Adverse events after radiofrequency ablation in patients with Barrett's esophagus: a systematic review and meta-analysis. Clin Gastroenterol Hepatol 2016; 14: 1086-1095.e6

[7] Gray NA, Odze RD, Spechler S]. Buried metaplasia after endoscopic ablation of Barrett's esophagus: a systematic review. Am J Gastroenterol 2011; 106: 1899-1908 quiz 1909

[8] Pimentel-Nunes P, Dinis-Ribeiro M, Ponchon T et al. Endoscopic submucosal dissection: European Society of Gastrointestinal Endoscopy (ESGE) Guideline. Endoscopy 2015; 47: 829-854

[9] Terheggen G, Horn EM, Vieth M et al. A randomised trial of endoscopic submucosal dissection versus endoscopic mucosal resection for early Barrett's neoplasia. Gut 2017; 66: 783-793

[10] Siddiki H, Fukami N. Endoscopic submucosal dissection for Barrett's neoplasia: decade of experience, little progress. Is ESD thE-BEST for complex Barrett's neoplasia? Gastrointest Endosc 2017; 86: 619-622

[11] Silva FB, Dinis-Ribeiro M, Vieth M et al. Endoscopic assessment and grading of Barrett's esophagus using magnification endoscopy and narrow-band imaging: accuracy and interobserver agreement of different classification systems (with videos). Gastrointest Endosc 2011; 73: 7-14

[12] Swager AF, Curvers WL, Bergman JJ. Diagnosis by endoscopy and advanced imaging of Barrett's Neoplasia. Adv Exp Med Biol 2016; 908: $81-98$

[13] Baldaque-Silva F, Marques M, Andrade AP et al. Endoscopic submucosal dissection of gastrointestinal lesions on an outpatient basis. United European Gastroenterol J 2019; 7: 326-334

[14] Holt BA, Bourke MJ. Wide field endoscopic resection for advanced colonic mucosal neoplasia: current status and future directions. Clin Gastroenterol Hepatol 2012; 10: 969-979
[15] Subramaniam S, Chedgy F, Longcroft-Wheaton G et al. Complex early Barrett's neoplasia at 3 Western centers: European Barrett's Endoscopic Submucosal Dissection Trial (E-BEST). Gastrointest Endosc 2017; 86: 608-618

[16] Neuhaus H, Terheggen G, Rutz EM et al. Endoscopic submucosal dissection plus radiofrequency ablation of neoplastic Barrett's esophagus. Endoscopy 2012; 44: 1105-1113

[17] Yamaguchi N, Isomoto H, Nakayama T et al. Usefulness of oral prednisolone in the treatment of esophageal stricture after endoscopic submucosal dissection for superficial esophageal squamous cell carcinoma. Gastrointest Endosc 2011; 73: 1115-1121

[18] Schlemper R], Riddell RH, Kato Y et al. The Vienna classification of gastrointestinal epithelial neoplasia. Gut 2000; 47: 251-255

[19] Shaheen NJ, Overholt BF, Sampliner RE et al. Durability of radiofrequency ablation in Barrett's esophagus with dysplasia. Gastroenterology 2011; 141: 460-468

[20] Altorki NK, Lee PC, Liss Y et al. Multifocal neoplasia and nodal metastases in T1 esophageal carcinoma: implications for endoscopic treatment. Ann Surg 2008; 247: 434-439

[21] Sato H, Inoue H, Kobayashi $\mathrm{Y}$ et al. Control of severe strictures after circumferential endoscopic submucosal dissection for esophageal carcinoma: oral steroid therapy with balloon dilation or balloon dilation alone. Gastrointest Endosc 2013; 78: 250-257

[22] Babyak MA. What you see may not be what you get: a brief, nontechnical introduction to overfitting in regression-type models. Psychosom Med 2004; 66: 411-421

[23] Shaheen NJ, Falk GW, lyer PG et al. American College of Gastroenterology. ACG Clinical Guideline: Diagnosis and Management of Barrett's Esophagus. Am J Gastroenterol 2016; 111: 30-50 quiz 51

[24] Weusten B, Bisschops R, Coron E et al. Endoscopic management of Barrett's esophagus: European Society of Gastrointestinal Endoscopy (ESGE) Position Statement. Endoscopy 2017; 49: 191-198

[25] Phoa KN, Pouw RE, Bisschops R et al. Multimodality endoscopic eradication for neoplastic Barrett oesophagus: results of an European multicentre study (EURO-II). Gut 2016; 65: 555-562

[26] Komeda Y, Bruno M, Koch A. EMR is not inferior to ESD for early Barrett's and EG] neoplasia: An extensive review on outcome, recurrence and complication rates. Endosc Int Open 2014; 2: E58-E64

[27] Probst A, Aust D, Märkl B et al. Early esophageal cancer in Europe: endoscopic treatment by endoscopic submucosal dissection. Endoscopy 2015; 47: 113-121

[28] Yang D, Coman RM, Kahaleh M et al. Endoscopic submucosal dissection for Barrett's early neoplasia: a multicenter study in the United States. Gastrointest Endosc 2017; 86: 600-607

[29] Goa KL, Benfield P. Hyaluronic acid. A review of its pharmacology and use as a surgical aid in ophthalmology, and its therapeutic potential in joint disease and wound healing. Drugs 1994; 47: 536-566

[30] Coman RM, Gotoda T, Forsmark CE et al. Prospective evaluation of the clinical utility of endoscopic submucosal dissection (ESD) in patients with Barrett's esophagus: a Western center experience. Endosc Int Open 2016; 4: E715-E721

[31] Chung A, Bourke M], Hourigan LF et al. Complete Barrett's excision by stepwise endoscopic resection in short-segment disease: long term outcomes and predictors of stricture. Endoscopy 2011; 43: 10251032

[32] Chevaux JB, Piessevaux H, Jouret-Mourin A et al. Clinical outcome in patients treated with endoscopic submucosal dissection for superficial Barrett’s neoplasia. Endoscopy 2015; 47: 103-112 